\title{
Mixed Mode Cohesive Crack Propagation
}

A. CARPINTERI, S. VALENTE and P. BOCCA Politecnico di Torino, Department of Structural Engineering, 10129 Torino, Italy.

\section{ABSTRACT}

A cohesive crack model is proposed to describe strain localization for the materials where strain-hardening is not prevailing over strain-softening (geomaterials, concrete-like materials, ceramics, etc.). Such a model is able to predict the size effects of fracture mechanics, i.e., the transition from ductile to brittle structure behaviour by increasing the size scale and keeping the geometrical shape unchanged.

Whereas for Mode $I$, only untieing of the finite element nodes is applied to simulate crack growth, for Mixed Mode interelement crack propagation a topological variation is required at each step. In the case of four point shear testing, the load vs. deflection diagrams reveal snap-back instability for large sizes. By increasing the specimen sizes, such instability tends to reproduce the classical LEFM instability, predicted by the Maximum Circumferential stress Criterion. Experimentally, the fracture toughness parameter of concrete appears to be unique and represented by the Mode I fracture energy $G_{F}$ or the stress-intensity factor $K_{I C}$, even for Mixed Mode problems.

\section{KEYWORDS}

Fracture mechanics; concrete; strain-softening; mode II; cohesive crack; snap-back; instability; brittleness number.

\section{INTRODUCTION}

According to the cohesive crack model, the non-linear crack behaviour can be described by means of cohesive forces in the process zone, representing 
plastic flow, aggregate interlocking, fiber bridging, etc. In this way, strain localization is taken into account for the materials where strainsoftening is prevailing over strain-hardening.

The cohesive crack model was originally proposed by Barenblatt (1959) and, independently, by Dugdale (1960). Later, it was reconsidered by Bilby, Cottrell and Swinden (1963), Willis (1967) and Rice (1968). More recently, the cohesive crack model was reproposed, with some modifications, by Wnu (1974) - the Final Stretch Model - and by Hillerborg, Modeer and Petersson (1976) - the Fictitious Crack Model. The latter was applied mostly to concrete-like materials and numerically implemented in a finite element program.

In the present paper, the cohesive crack model is applied to analyze the stable versus unstable crack propagation in elastic-softening materials. The shape of the structural load-displacement response changes substantially by varying size-scale and keeping geometrical shape of the structure unchanged. For size-scales larger than a threshold value, a snap-back instability appears, when the plastic zone is still absent and the slow crack growth has not occurred yet. Asymptotically, the snap-back load may be provided by the simple LEFM condition: $K_{I}=K_{I C}$ in Mode $I$, or by the Maximum Circumferential Stress Criterion (Erdogan and $\operatorname{Sih}, 1963): F\left(K_{I}, K_{I I}\right)=K_{I C}$, in Mixed Mode.

The size-scale transition from ductile to brittle behaviour is governed by dimensionless brittleness number $\mathrm{s}_{\mathrm{E}}$, which is a function of material properties and structure size.

The snap-back load-deflection branch may be captured experimentally only if the loading process is controlled by a monotonically increasing function of time, e.g. the crack mouth opening or sliding displacement. On the other hand, the snap-back load-deflection branch may be captured numerically only if the loading process is controlled by a monotonically increasing function of the crack length. An example of such function is provided by the $n$ indirect displacement control scheme" (Rots and de Borst, 1987). This technique uses a displacement norm as controlling parameter. On the other hand, as monotonically increasing function of the crack length, it is possible to use the crack length itself, in Mode I (Carpinteri, 1985; Carpinteri and Fanelli, 1987) as well as in Mixed Mode (Carpinteri and Valente, 1988; Bocca, Carpinteri and Valente, 1988). Such technique, called "crack length contro schemen, will be proposed in the present paper. FEM-crack propagation requires a continuous modification of the mesh. Whereas for Mode $I$, only node untieing is applied to simulate crack growth, for Mixed Mode interelement crack propagation a topological variation must be performed at each step automatically (Wawrzynek and Ingraffea, 1987).
The amount of energy dissipated in the localized Mixed Mode fracture zone results to be experimentally equal to the product of Mode I fracture energy resule the introduction of an additional $\mathrm{G}_{\mathrm{F}}$ and total fracture area. Therefore, the introdess unnecessary. fracture toughness parameter for Mixed Mode problens, appears und The assumption of the "Maximum Circumferential Stress Criterion", for which any crack growth step is produced by a Mode I (or opening) mechanism, seems to be confirmed by the experimental results.

\section{COHESIVE CRACK MODEL}

The Principlef Vork can be used as the integral statement to formulate the elastic-softening problem in terms of finite element approximation:

$$
\int_{V} d \varepsilon^{T} \sigma d v=\int_{V} d u^{T} F d V+\int_{S} d u^{T} p d S
$$

where $\sigma^{T}=\left[\sigma_{x}, \sigma_{y}, \sigma_{z}, \tau_{x y}, \tau_{y z}, \tau_{x z}\right]$ is the stress vector, $d \varepsilon^{T}$ is the vector of incremental virtual strain $F^{T}=\left[F_{X}, F_{y}, F_{z}\right]$ is the vector of body forces acting per unit volume, $d u^{T}=[d u, d v, d w]$ is the vector of in body forces acting ${ }^{T}=\left[p_{x}, p_{y}, p_{z}\right]$ is the vector of tractions acting per unit area of external surface $s$.

Eg. (1) is the weak form of the equilibrium equations and is valid for linear as well as for non-linear stress-strain constitutive laws.

According to the cohesive crack model, the process zone near the crack tip According the crack the last term in eq.(1) can be decomposed as follows (Fig.1):

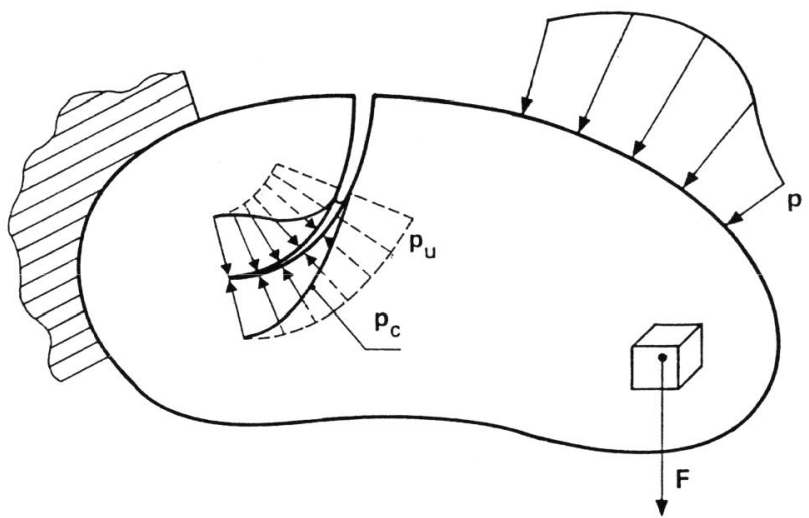

Fig.1. Mixed Mode cohesive crack propagation. 


$$
\int_{S} d u^{T} p d S=\int_{S_{C}} d u^{T} p_{C} d S+\int \underset{S-S_{C}}{d u^{T} p d S}
$$

where $S_{C}$ is the process zone, i.e., the crack surface where the cohesive forces are active.

Assuming a linear softening constitutive law, the traction versus displacement relationship can be written (Fig.1):

$$
p_{C}=p_{u}+N^{T} L N\left(u^{+}-u^{-}\right)
$$

where $\mathrm{pu}_{\mathrm{u}}$ is the ultimate tensile strength in vectorial form, $\mathbf{N}$ is the transformation matrix from the global to the local reference system, varying point by point on the crack surface, $L$ is the cohesive constitutive matrix in a local cartesian system, the index + refers to the positive side of the crack, while the index - refers to the negative one.

From equilibrium considerations across the crack surface, it is possible to write:

$$
\mathrm{p}_{\mathrm{c}}^{+}=-\mathrm{p}_{\mathrm{c}}^{-}, \mathrm{p}_{\mathrm{u}}^{+}=-\mathrm{p}_{\mathrm{u}}^{-}, \mathrm{s}_{\mathrm{c}}^{+}=\mathrm{s}_{\mathrm{c}}^{-}=\mathrm{s}_{\mathrm{c}} / 2 .
$$

The first term in the right-hand side of eq.(2) can be written:

$$
\begin{aligned}
& \int_{S_{C}} d^{T} p_{C} d S=\int_{S_{C}^{T}}^{q u^{+T}} p_{u}^{+} d S+\int_{S_{C}^{-}} u^{-T} p_{u}^{-} d S+ \\
& +\int_{S_{C}} \underset{u^{+T}}{+T} N^{T} L N\left(u^{+}-{u^{-}}^{-}\right) d S-\int_{S_{C}^{-}} d u^{-T} N^{T} L N\left(u^{+}-u^{-}\right) d S .
\end{aligned}
$$

The last two terms in eq. (5) can be represented as follows:

$$
\int_{S_{C} / 2}\left\{\begin{array}{l}
d^{+} \\
d u^{-}
\end{array}\right\}^{T}\left[\begin{array}{ll}
N^{T} & 0 \\
0 & N^{T}
\end{array}\right]\left[\begin{array}{lr}
L & -L \\
-L & L
\end{array}\right]\left[\begin{array}{ll}
N & 0 \\
0 & N
\end{array}\right]\left\{\begin{array}{l}
u^{+} \\
u^{-}
\end{array}\right\} d s .
$$

The Principle of Virtual Work, eq.(1), can be developed according to eqs $(2),(5)$ and (6):

$$
\begin{aligned}
& \int_{V} d \varepsilon^{T} \sigma d V=\int_{V} d u^{T} F d V+\int_{S-S_{C}} d u^{T} p d S+\int_{S_{C}^{+}} d u^{+T} p u d S+\int_{S_{C}^{-}} d u^{-T} p \bar{u} d S+ \\
& +\int_{S_{C} / 2}\left\{\begin{array}{l}
d u^{+} \\
d u^{-}
\end{array}\right\}^{T}\left[\begin{array}{rr}
N^{T} & 0 \\
0 & N^{T}
\end{array}\right]\left[\begin{array}{rr}
L & -L \\
-L & L
\end{array}\right]\left[\begin{array}{ll}
N & 0 \\
0 & N
\end{array}\right]\left\{\begin{array}{l}
u^{+} \\
u^{-}
\end{array}\right\} d S .
\end{aligned}
$$

Subdividing the domain in a finite number of elements and expressing the internal displacements by means of locally based shape functions $H$, it is possible to write:

$$
u(x, y, z)=H(x, y, z) u .
$$

From derivation of eq.(8), the strain versus displacement relationship can be obtained:

$$
\varepsilon=B \text { u. }
$$

Selecting an appropriate constitutive law for the uncracked zone, the stress versus strain relationship appears as follows:

$$
\sigma=D\left(\varepsilon-\varepsilon_{0}\right)+\sigma_{0} \text {. }
$$

Substituting eqs $(8),(9)$ and $(10)$ in eq. (7), and indicating by "e" the generic element, it is possible to write:

$$
\begin{aligned}
& d u^{T}\left(\sum_{e} \int_{V} B^{T} D B d v\right) u-\left\{\begin{array}{l}
d u^{+} \\
d u^{-}
\end{array}\right\} \text {T }\left(\sum_{e} \int_{S_{C^{\prime}} / 2} H^{T}\left[N^{T}\right][L][N] H d S\right)\left\{\begin{array}{l}
u^{+} \\
u^{-}
\end{array}\right\}= \\
& =d u^{T} \sum_{e} \int_{V}\left(H^{T} F-B^{T} \sigma_{0}+B^{T} D \varepsilon_{0}\right) d V+ \\
& +d u^{T}\left(\sum_{e} \int_{S-S_{C}}^{H^{T}} p d S\right)+d u^{+T}\left(\sum_{e} \int_{S^{+}}^{H^{T}} P_{u}^{+} d S\right)+d u^{-T}\left(\sum_{e} \int_{S^{-}}^{H^{T}} P_{u}^{-} d S\right) .
\end{aligned}
$$

Since :

$\left\{u^{+}\right\} \subset\{u\},\left\{u^{-}\right\} \subset\{u\},\left\{d u^{+}\right\} \subset\{d u\},\left\{d u^{-}\right\} \subset\{d u\}$,

an assemblage procedure can be carried out:

$$
(K-C) \quad u=F_{v}+F_{S}+F_{u}^{+}+F_{u^{-}}^{-},
$$

where:

$$
\begin{aligned}
& K=\text { stiffness matrix, } \\
& C=\text { softening matrix, } \\
& F_{V}, F_{S}, F_{U}^{+}, F_{u}^{-}=\text {loading vectors, } \\
& (K-C)=\text { effective stiffness matrix. }
\end{aligned}
$$

Neglecting the tangential cohesive tractions, the constitutive matrix L becomes: 


$$
\mathbf{L}=\left[\begin{array}{ccc}
0 & 0 & 0 \\
0 & 0 & 0 \\
0 & 0 & 133
\end{array}\right]
$$

Only the component of the mutual displacement normal to the crack surface, $w$ (crack opening displacement), is taken into account. The remaining components are disregarded.

The scalar quantity $1_{33}$ is assumed as follows:

$$
\begin{array}{ll}
1_{33}=\frac{\sigma_{u}}{w_{C}}, & \text { for } 0<w<w_{C} \\
l_{33}=0, & \text { for } w \geq w_{C}
\end{array}
$$

where $\sigma_{u}$ is the ultimate tensile strength of the material and $w_{C}$ is the critical value of the crack opening displacement $w$. For crack opening displacements greater than the critical value $w_{C}$, the interaction forces disappear, and both the crack surfaces are stress-free. During the irreversible fracture process, the crack opening displacement $w$ results to be a monotonic increasing function of time.

At the first step the cohesive zone is absent, matrix $C$ vanishes and matrix $K$ is positive definite. A linear elastic solution can be found, giving position and orientation of the growing crack. The crack surface $s_{C}$ starts propagating by a pre-defined length $\Delta S_{C}$. Such an incremental length is chosen so small that matrix $(K-C)$ renains positive definite, and the maximum cohesive crack opening displacement is less than $w_{C}$. Eq.(13) can be solved for two right-hand side vectors:

$$
\begin{aligned}
& (K-C) u_{1}=F_{v}+F_{S}, \\
& (K-C) u_{2}=-F_{u^{+}}{ }^{+}-F_{u^{-}} .
\end{aligned}
$$

If $\sigma_{\theta}$ is the circumferential stress at the fictitious crack tip, for each value of the angle $\theta$ it is possible to write:

$$
\lambda\left(\sigma_{\theta}\right)_{1}-\left(\sigma_{\theta}\right)_{2}=\sigma_{u},
$$

and then, solving with respect to the loading multiplier $\lambda$ :

$$
\lambda=\frac{\sigma_{u}+\left(\sigma_{\theta}\right)_{2}}{\left(\sigma_{\theta}\right)_{1}} \text {. }
$$

Eq.(18) is interpretable as a function $\lambda=\lambda(\theta)$. The minimum of $\lambda=\lambda(\theta)$. and the related displacement vector:

$$
u=\lambda(\min ) u_{1}-u_{2},
$$

describe the second step of the cracking process, providing the orientation of the subsequent crack branch.

at the following steps the same procedure is repeated, without moving the At the following conditions is verified.

(1) (1) The crack openir

cal value $w_{C}$.

(2) Matrix (K-C) in eq.(16) becomes positive semidefinite.

In both cases, the real crack tip moves and the cohesive crack surface $s_{c}$ In both cack tip is less than $w_{C}$, or, respectively, matrix $(K-C)$ is positive definite.

\section{SNAP-BACK SOFTENING INSTABILITY AND BRITTLE MIXED MODE FRACTURES .}

Por Mixed Mode fracture, a topological variation is required at each step of For the interelenent crack model. shear specimen (Fig.2) is analyzed according to thlowing: $1=4 b, t=b$, The geometrical features of the specimen are the the material is assumed to

$\mathrm{a}_{0}=0.2 \mathrm{~b}, \mathrm{c}=0.8 \mathrm{~b}$ and $0.4 \mathrm{~b}$, whereas the material is ass
present the ultimate strain $\varepsilon_{\mathrm{u}}=\sigma_{\mathrm{u}} / \mathrm{E}=0.741 \times 10^{-4}$ and $\nu=0.1$.

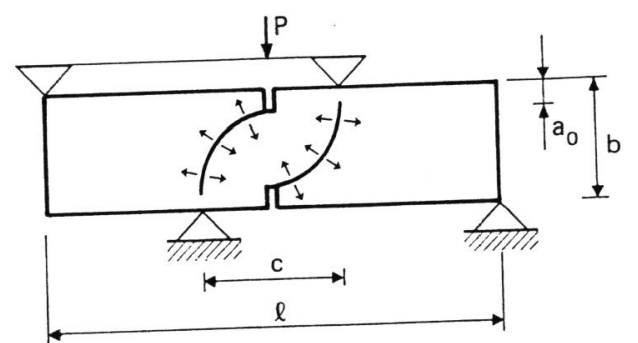

Fig.2. Four point shear specimen.

The dimensionless load versus deflection diagrams in Fig.3-a are related to The dimensionless load the larger distance between the contrer distance $(c / b=0.4)$. Both diagrams in Fig.3-b are referring to the smaller distance being:

$$
s_{E}=G_{I C} / \sigma_{u} b, G_{I C}=\int_{0}^{w} c \text { } \sigma(w) d w .
$$


On the other hand, an evident difference in the p- $\delta$ shape transpires. Whereas for $c / b=0.8$, the snap-back branch ( $d P / d \delta>0$ ) is followed by normal softening tail ( $\mathrm{dP} / \mathrm{d} \delta<0$ ), passing through the stationary condition $\mathrm{d} \delta / \mathrm{dP}=0$, for $\mathrm{c} / \mathrm{b}=0.4$ the normal softening tail does not appear after the snap-back behaviour, and the snap-back branch tends to go back to the origin. The latter kind of equilibrium path reveals a potentially brittler behaviour. In fact, if the loading process is deflection-controlled,
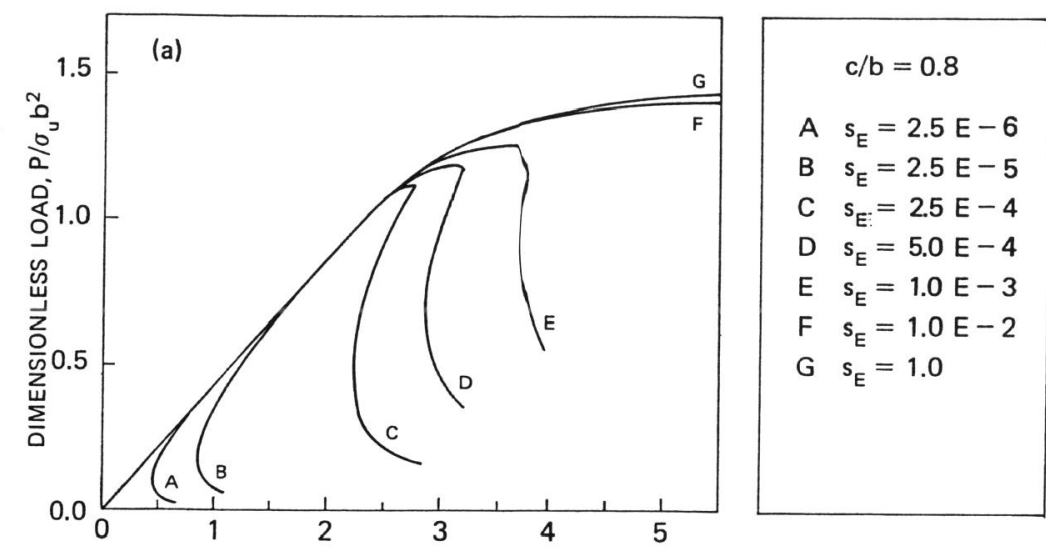

DIMENSIONLESS DEFLECTION, $\delta / \mathrm{b} \times 10^{4}$

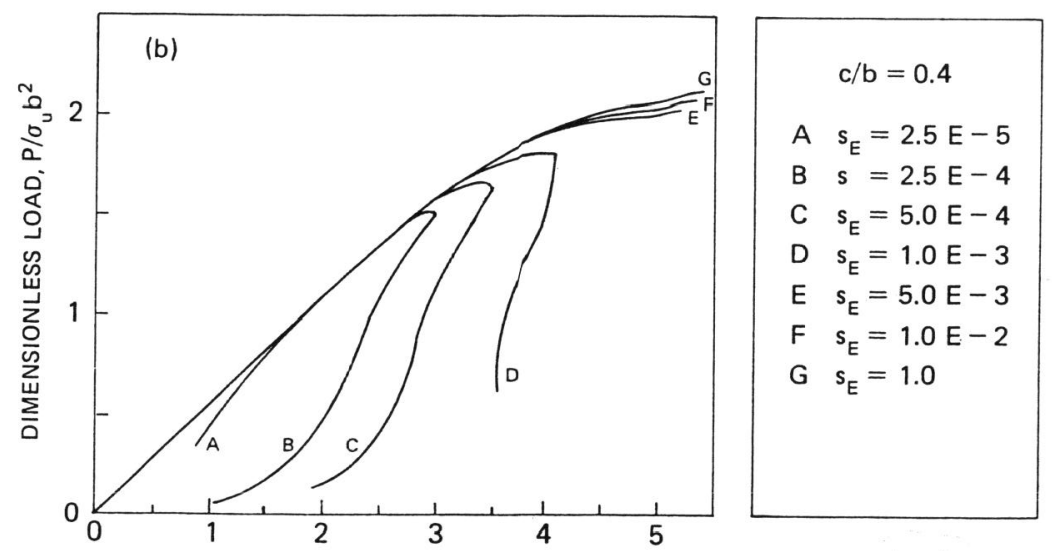

DIMENSIONLESS DEFLECTION, $\delta / \mathrm{b} \times 10^{4}$

Fig. 3. Ductile-brittle transition by varying the brittleness number $s_{E}=G_{I C} / \sigma_{u} b$

(four point shear specimen).

(a) $c / b=0.8 ;$ (b) $c / b=0.4$. the loading capacity would show a vertical drop down to zero, without any possibility for the crack to arrest.

The maximum loading capacity $\mathrm{P}_{\mathrm{COHES}}$ according to the cohesive crack model, is provided by the diagrams in Fig.3 . On the other hand, the maximum load Circumferential Stress Criterion (Erdogan and Sih, 1963):

$$
\frac{d \sigma_{\theta}}{d \theta}=0, \quad \sigma_{\theta} \sqrt{2 \pi r}=k_{I C}=\sqrt{G_{I C} E} .
$$

Stress intensification is produced in both the crack tip regions and the stress-intensity factors for Mode I and Mode II can be expressed respectively as:

$$
\begin{aligned}
K_{I} & =\frac{P}{t b^{1 / 2}} f_{I}\left(\frac{l}{b}, \frac{a}{b}, \frac{c}{b}\right), \\
K_{I I} & =\frac{P}{t b^{1 / 2}} f_{I I}\left(\frac{1}{b}, \frac{a}{b}, \frac{c}{b}\right),
\end{aligned}
$$

$f_{I}$ and $f_{I I}$ being the shape functions.

The angle $\theta_{0}$ of crack branching is provided by the following equation:

$$
f_{I} \sin \theta_{0}+f_{I I}\left(3 \cos \theta_{0}-1\right)=0 \text {, }
$$

whereas the Mixed Mode crack instability is predicted by the condition:

$$
P_{\text {LEFM }} \cos \frac{\theta_{0}}{2}\left[f_{I} \cos ^{2} \frac{\theta_{0}}{2}-\frac{3}{2} f_{I I} \sin \theta_{0}\right]=t b^{1 / 2} K_{I C} .
$$

The values of the ratio $\mathrm{P}_{\text {COHES }} / \mathrm{P}_{\text {LEFM }}$ are represented in Fig. 4 against the dimensionless size $1 / \mathrm{s}_{\mathrm{E}}$. A transition is evident towards LEFM by increasing the size-scale of the structure. For the brittler geometry, $c / b=0.4$, the transition appears to be faster, and already for $b \sigma_{u} / G_{I C}=2 \times 10^{4}$ or $S_{E}=5 \times 10^{-5}$, the asymptotical LEFM condition is achieved. In this case, the size of the cohesive zone is negligible with respect to the size of the zone where the $\mathrm{r}^{-1 / 2}$ LEFM-stress-singularity is dominant.

For $c / b=0.8$, the total load versus loading point deflection diagrams are plotted in Figs, $5-a$ and $b$, in the cases $b=5$ and $20 \mathrm{~cm}$ respectively. The Mixed Mode cohesive crack model describes both the experimental curves obtained in (Bocca, Carpinteri and Valente, 1988) satisfactorily. The size $b=20 \mathrm{~cm}$ (Fig.5-b) produces snap-back instability in the experimental as well as in the numerical curve. The mechanical properties utilized in the numerical analysis are: Young's modulus $\mathrm{E}=27000 \mathrm{MPa}$, ultimate tensile strength $\sigma_{\mathrm{u}}=2 \mathrm{MPa}$, fracture energy $\mathrm{G}_{\mathrm{F}}=100 \mathrm{~N} / \mathrm{m}$. 


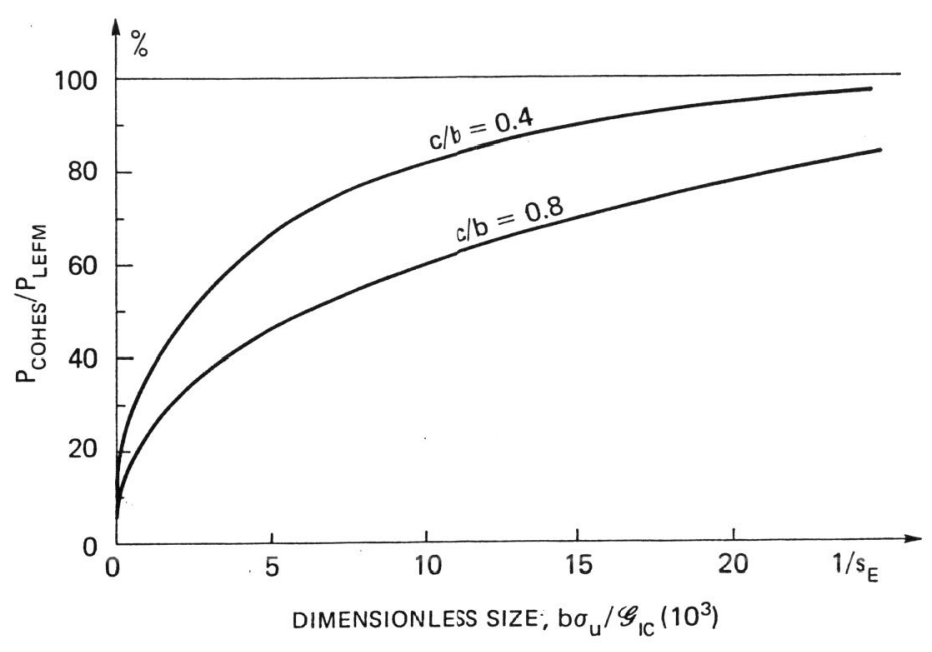

Fig.4. Size-scale transition towards Mixed Mode-LEFM-instability.

The area enclosed between numerical curve and deflection axis is approximately equal to the product of the Mode I fracture energy $G_{F}$ and the total fracture area, and represents the amount of energy dissipated in the localized fracture zone. The amount of energy dissipated by punching at the supports, was deliberately neglected, assuming ascending elastic branches consistent with the elastic modulus of the material (Figs.5).

It is remarkable that the application of the usual Mode I fracture energy $G_{F}$ only, was able to provide consistent results. It was unnecessary to introduce additional fracture toughness parameters, like, for example, the Mode II fracture energy $G_{F} I I$ (Rots and de Borst, 1987; Bažant and Pfeiffer, 1986). As a matter of fact, the Mixed Mode fracture energy results approximately equal to the Mode I fracture energy $G_{F}$, each elementary crack growth step being produced by a Mode I (or opening) mechanism along the curvilinear trajectory.

The sequence of the finite element meshes utilized for the case $b=20 \mathrm{~cm}$, $\mathrm{c} / \mathrm{b}=0.8$, is reported in Fig.6. The trajectory of the finite element rosette reproduces the experimental fracture trajectory accurately. It is remarkable that the real crack (complete disconnection) starts propagating only at the 13th step, when the fictitious crack (cohesive interaction) is beyond one half of the beam depth. On the other hand, at the 22 th step, both fictitious and real crack are close to the upper beam edge. The single steps are indicated also in the diagram of Fig.5-b.
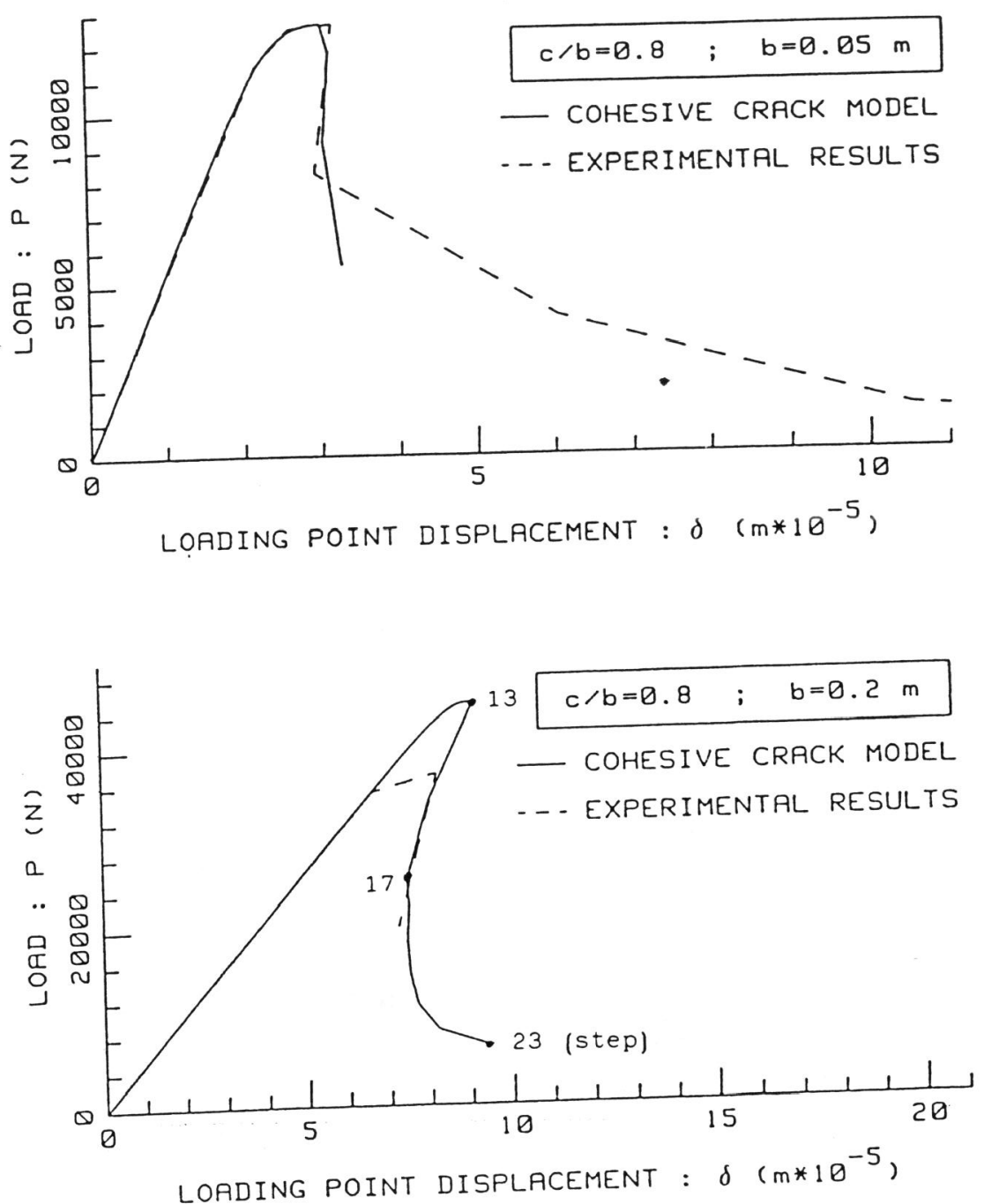

Fig. 5. Experimental load vs. deflection curves and numerical cohesive crack simulation, for $c / b=0.8$. (a) $b=5 \mathrm{~cm}$; (b) $b=20 \mathrm{~cm}$ 

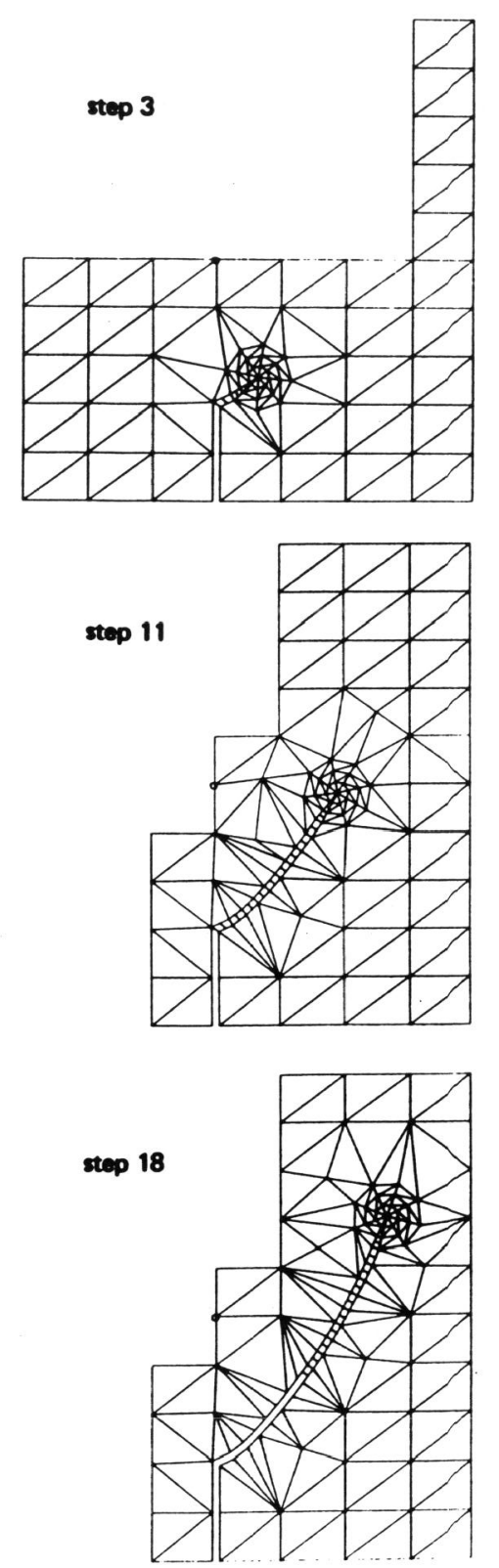
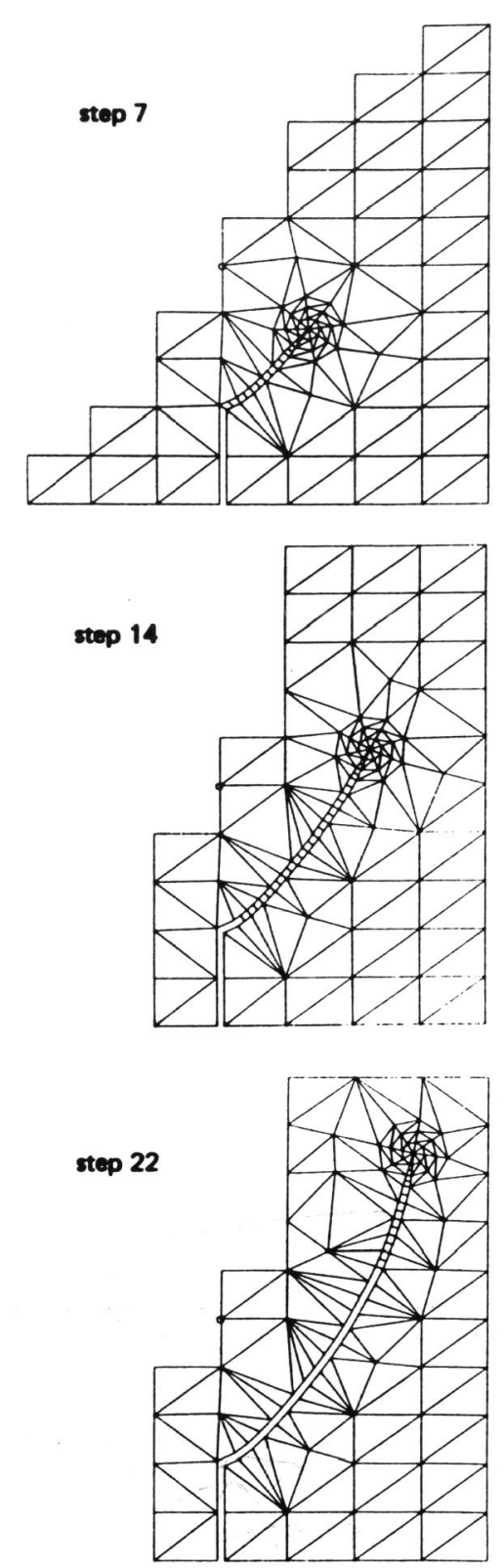

Fig.6. Finite element remeshing. $\mathrm{c} / \mathrm{b}=0.8 ; \mathrm{b}=20 \mathrm{~cm}$
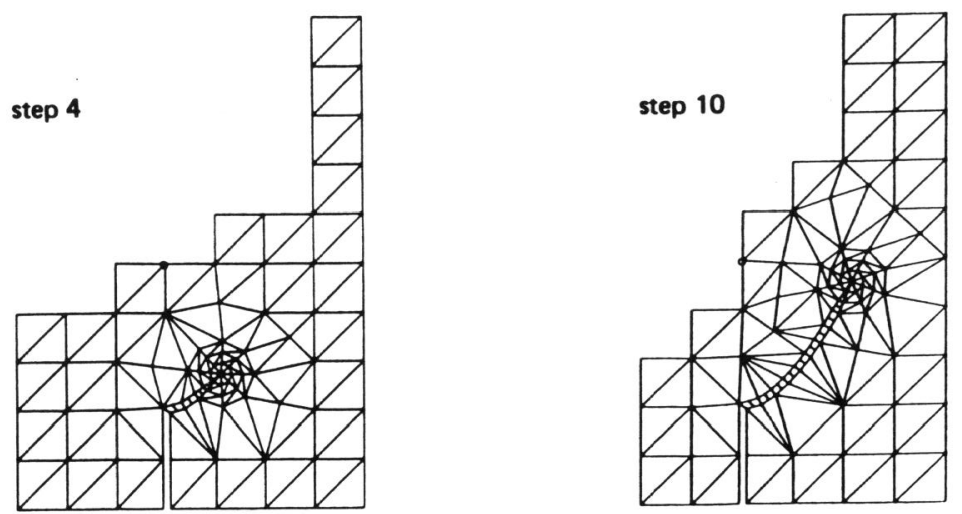

step 14

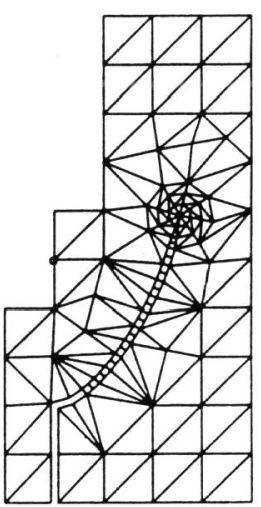

step 22

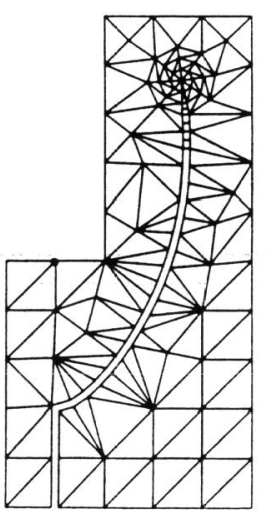

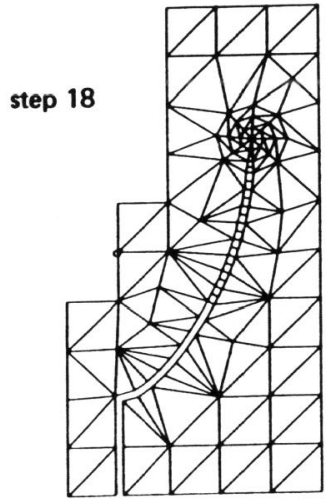

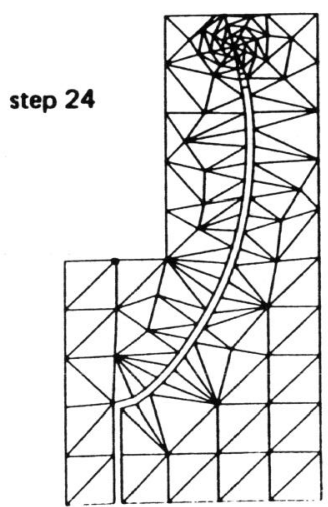

Fig.7. Finite element remeshing. $c / b=0.4 ; b=20 \mathrm{~cm}$ 
The sequence of the finite element meshes utilized for the case $b=20 \quad c m$, $c / b=0.4$, is reported in Fig.7. Also in this case, the numerical simulation describes the experimental fracture trajectory very accurately, included the deviations at the beam edges shown in Fig.8 (Bocca, Carpinteri and Valente, 1988)

\section{ACKNOWLEDGEMENTS}

The financial support of the Department of Public Education (M.P.I.) is gratefully acknowledged.

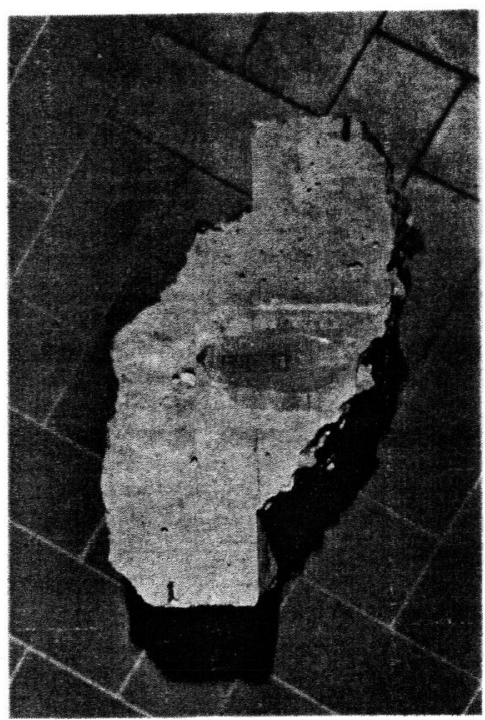

Fig. 8. Experimental trajectories followed by the two symmetrical cracks in the case $c / b=0.4$, $\mathrm{b}=20 \mathrm{~cm}$.
Barenblatt, G.I. (1959). The formation of equilibrium cracks during brittle fracture: general ideas and hypotheses. Axially-symmetric cracks. Journal fracture:

of Applied Mathematics and Mechanics, 23 . Shear fracture tests of concrete, Materials and Structures, 19, 111-121.

(1963). The spread of plastic yield from a notch, Proc. R. Soc., A272, 304-314.

yield from a notch, Bocca, P., Carpinteri, A. and Valente, S. (1988). Size effects in the mixed mode crack propagation: softening and snap-back and Rock, Vienna (Austria), Conference on Frac

July 4-6, 1988.

Carpinteri, A. (1984). Interpretation of the Griffith instability as a bifurcation of the global equilibrium. NATO Advanced Research Workshop on Application of Fracture Mechanics to Cementitious Composites, Evanston (Illinois),

Panteri, A. and Fanell, M. (1987). Numerical analysis of the catastrophical softening behaviour in brittle structures. Fourth International Pan Antonio (Texas), March 23-27, 1987, Pineridge Press, pp.369-386.

Carpinteri, A. and Valente, s. (1988). Numerical modelling of mixed mode cohesive crack propagation. International Conference on Computational Engineering Science, Atlanta (Georgia), April 10-14, 1988, S.N. Atluri and G. Yagawa, Eds, Springer-Verlag, pp.12-VI.

Dugdale, D.s. (1960) Yielding of steel sheets containing slits. Journal of Mechanics and Physics of Solids, $\underline{8}, 100-104$

Erdogan, F. and Sih, G.C. (1963). On the crack extension in plates under plane lournal of Basic Engineering, 85, 519527

Hillerborg, A., Modeer, M. and Petersson, P.E. (1976). Analysis of crack formation and crack growth in concrete by means of fracture mechanics and finite

(1968). A path independent integral and the approximate analysis of strain concentration by notches and cracks. J. Appl. Mech., 35, 379386.

Rots. J and de Borst, R. (1987). Analysis of mixed mode fracture in concrete, Journal of Engineering Mechanics, 113, 1739-1758.

Wrzynek, P.A., and Ingraffea, A.R. (1987). Interactive finite element analysis of fracture processes: An integrated approach, Theor. \& Appl. Frac. Mech., 8 , pp.137-150.

Mech., $\frac{8}{\text {, pp.137-150. }}$. Darnal of Mechanics and Physics of Solids, 15, 151-162. Bastic extension of a tensile crack contained in a viscoelastic-plastic solid. Journal of Applied Mechanics, 41, 234-242. 